\title{
Doença de Chagas: aspectos clínicos, epidemiológicos e fisiopatológicos
}

Chagas disease: clinical, epidemiological and pathophysiological aspects

Enfermedad de Chagas: aspectos clínicos, epidemiológicos y fisiopatológicos

Jennifer Rodrigues Correia ${ }^{1 *}$, Suzana Cristina Silva Ribeiro ${ }^{1}$, Lorena Vieira Fernandez de Araújo ${ }^{1}$, Mariane Costa Santos ${ }^{1}$, Thiago Reis Rocha ${ }^{2}$, Emanuelle Almeida Silva Viana ${ }^{1}$, Poliana Terra Pires Ribeiro Coelho Caires ${ }^{1}$, Shesllen Mikaelly Cruz Corrêa ${ }^{1}$, Taise Gonçalves Pinheiro ${ }^{1}$, Lenise Costa de Carvalho ${ }^{3}$.

\section{RESUMO}

Objetivo: Realizar uma revisão bibliográfica acerca das principais características epidemiológicas, clínicas e fisiopatológica da doença de Chagas. Revisão bibliográfica: Foi realizada uma análise bibliográfica nas bases de dados vinculadas à Biblioteca Virtual em Saúde, Pubmed e Acervo+. A doença de chagas é provocada pelo protozoário Trypanosoma cruzi e é considerada problema de saúde pública e social, pois, é caracterizada como parte da identidade da pobreza e dos maus tratos as condições de saúde, afetando populações em situação de vulnerabilidade, especialmente aquelas com baixa visibilidade e pouca voz política, desencadeando processos de estigma e discriminação. Ademais, é uma afecção que provoca impacto de morbimortalidade importante e é relativamente negligenciada por pesquisadores. ocorre de forma preferencial em homens e em zona rural na faixa etária entre 19 a 59 anos de idade. Considerações finais: Salientamos a importância da educação em saúde para informar a população acerca da transmissão da doença e dos cuidados referentes a prevenção. Ainda, é necessário que novos estudos sejam realizados, especialmente em pacientes idosos maiores de 60 anos de idade, tendo em vista a maior importância da infecção nesta faixa etária associada a comorbidades.

Palavras-chave: Doença de Chagas, Doenças negligenciadas, Vulnerabilidade social.

\begin{abstract}
Objective: To perform a bibliographic review on the main epidemiological, clinical and pathophysiological characteristics of Chagas disease. Bibliographic review: A bibliographic analysis was carried out on the databases linked to the Virtual Health Library, Pubmed and Collection +. Chagas disease is caused by the protozoan Trypanosoma cruzi and is considered a public and social health problem, as it is characterized as part of the identity of poverty and mistreatment of health conditions, affecting vulnerable populations, especially those with low visibility and little political voice, triggering processes of stigma and discrimination. Furthermore, it is a condition that has an important impact on morbidity and mortality and is relatively neglected by researchers. it occurs preferentially in men and in rural areas in the age group between 19 and 59 years of age. Final considerations: We emphasize the importance of health education to inform the population about the transmission of the disease and care related to prevention. Still, it is necessary that further studies are carried out, especially in elderly patients over 60 years of age, in view of the greater importance of infection in this age group associated with comorbidities.
\end{abstract}

Key words: Chagas disease, Neglected diseases, Social vulnerability.

\section{RESUMEN}

Objetivo: Realizar una revisión bibliográfica sobre las principales características epidemiológicas, clínicas y fisiopatológicas de la enfermedad de Chagas. Revisión bibliográfica: Se realizó un análisis bibliográfico de

\footnotetext{
${ }^{1}$ Faculdades Santo Agostinho (FASA), Vitória da Conquista - BA. *E-mail: jenniferrcc7@gmail.com

${ }^{2}$ Hospital Geral de Ipiaú, Ipiaú - BA.

${ }^{3}$ Centro Universitário UniFTC, Salvador - BA.
}

SUBMETIDO EM: 1/2021 
las bases de datos vinculadas a la Biblioteca Virtual en Salud, Pubmed y Collection +. La enfermedad de Chagas es causada por el protozoo Trypanosoma cruzi y es considerada un problema de salud pública y social, ya que se caracteriza como parte de la identidad de la pobreza y el maltrato de las condiciones de salud, afectando a poblaciones vulnerables, especialmente aquellas con poca visibilidad y poca voz política. desencadenando procesos de estigma y discriminación. Además, es una condición que tiene un impacto importante en la morbilidad y la mortalidad y es relativamente desatendida por los investigadores. ocurre preferentemente en hombres y en áreas rurales en el grupo de edad entre 19 y 59 años. Consideraciones finales: Destacamos la importancia de la educación en salud para informar a la población sobre la transmisión de la enfermedad y los cuidados relacionados con la prevención. Aún así, es necesario que se realicen más estudios, especialmente en pacientes mayores de 60 años, dada la mayor importancia de la infección en este grupo de edad asociada a comorbilidades.

Palabras clave: La enfermedad de Chagas, Enfermedades desatendidas, Vulnerabilidad social.

\section{INTRODUÇÃO}

Há mais de 100 anos o Trypanosoma cruzi foi identificado como o causador da doença de Chagas, mas até hoje a condição ainda se faz presente como um problema de saúde social e pública na América Latina (PÉREZ-MOLINA JA e MOLINA I, 2018). A doença de Chagas tem sido vista como parte da identidade de pobreza e maus tratos às condições de subsistências mínimas (PEREIRA LS, et al., 2015).

Afeta populações com baixa visibilidade e pouca voz política, causa estigma e discriminação, é relativamente negligenciada pelos pesquisadores e tem um considerável impacto na morbimortalidade e em despesas públicas em cuidados paliativos (MAGARAKIS M, et al., 2018; KRATZ MJ, et al., 2018).

De acordo com a Organização Mundial da Saúde (2019), estima-se que 6 a 7 milhões de pessoas estejam infectadas com o Trypanosoma cruzi, parasita causador da doença de Chagas (DIAS JCP, et al., 2016). Descoberta e descrita pelo pesquisador Carlos Chagas em 1909, trata-se de uma infecção transmissível que pode provocar lesões no miocárdio e no trato digestivo (VELOSO HH, et al., 2016).

Acredita-se que até $30 \%$ e $10 \%$ das pessoas infectadas irão desenvolver alterações cardíacas e digestivas, respectivamente (MARTINS-MELO FR, et al., 2012; PÉREZ-MOLINA JA e MOLINA I, 2018). Por muito tempo esquecida, a doença de Chagas está entre as 17 doenças tropicais mais negligenciadas de acordo com a OMS, o que remete a baixa campanha preventiva contra os vetores de contaminação, principalmente ao barbeiro, inseto responsável pela transmissão da maioria dos casos (CAVALCANTI MAF, et al., 2019).

Também conhecida como tripanossomíase americana, sua prevalência é maior nos países endêmicos da América Latina, sendo considerada uma doença potencialmente fatal, com sintomas e sinais que se subdividem em duas fases: aguda e crônica (ÁLVAREZ-HERNÁNDEZ DA, et al., 2018).

A fase aguda que perdura por até 2 meses, pode ser assintomática ou ainda manifestar-se por meio de febre, dores de cabeça, aumento das glândulas linfáticas e palidez. Já na fase crônica, os parasitas estão ocultos no coração e na musculatura digestiva (ORTIZ JV, et al., 2018).

A infecção pode levar a morte súbita por diminuição da função cardíaca, por destruição da sua musculatura e do sistema nervoso (PÉREZ-MOLINA JA e MOLINA I, 2018). Tratando-se do cenário brasileiro, destaca-se dentre as doenças crônicas por acometer cerca de 1,2 a 3 milhões de indivíduos e por configurarse como a quarta causa de morte entre as doenças infectoparasitárias nas faixas etárias acima dos 45 anos, representando 21.000 mortes/ano (COSTA AC, et al., 2018).

Mediante ao ônus, a prevenção, o controle e o tratamento da doença de Chagas tornaram-se prioridade para as entidades de fomento e financiamento da saúde local e mundial, elencando-a como um problema de saúde pública emergente, retomando os pontos importantes de prevenção e controle da doença de Chagas (CONNERS EE, et al., 2016). Diante do exposto, o presente constructo teve como objetivo realizar uma revisão bibliográfica acerca das principais características epidemiológicas, clínicas e fisiopatológica da doença de chagas. 


\section{REVISÃO BIBLIOGRÁFICA}

A produção de fatores de virulência por Trypanosoma cruzi durante a fase aguda inibe fortemente a resposta do sistema imunológico do hospedeiro, induzindo anergia e exclusão clonal de linfócitos $\mathrm{T}$, além de uma forte estimulação policlonal de linfócitos $B$ que secretam anticorpos com baixa afinidade para Antígenos de Trypanosoma cruzi. Isso promove a persistência da infecção e sua progressão para a fase crônica da doença (HABERLAND A, et al., 2013).

Na fase crônica, os mecanismos por trás da transição da fase assintomática para a fase sintomática ainda não foram totalmente elucidados. No entanto, acredita-se que haja muitos fatores envolvidos, como diferenças entre cepas de Trypanosoma cruzi, carga parasitária, tempo de infecção, antecedentes genéticos e resposta imune do hospedeiro (GRAYSON M, 2010; KRATZ MJ, et al., 2018).

Algumas teorias tentaram explicar o processo fisiopatológico da doença, incluindo: teoria da persistência do parasita (a presença e replicação de amastigotas nas células hospedeiras causam ruptura mecânica e secreção de resíduos que atraem células pró-inflamatórias); teoria neurogênica unificada (a perda significativa de neurônios no sistema nervoso simpático e parassimpático não está relacionada à presença de Trypanosoma cruzi in situ e é atribuída à produção e liberação de uma neurotoxina de um ninho de parasita escondido no corpo do hospedeiro) (BENCK L, et al., 2018).

Há ainda, a teoria autoimune (interação citotóxica acelerada que existe entre linfócitos relacionados à resposta imune ao Trypanosoma cruzi e miocardiócitos halogênicos não infestados por parasitas). Cada teoria apresenta discrepâncias únicas, o que pode ser explicado do ponto de vista clínico pela dificuldade em determinar a patogenicidade após um período prolongado entre a infecção pelo Trypanosoma cruzi e o desenvolvimento de suas complicações (BENCK L, et al., 2018; CONNERS EE, et al., 2016).

A fase aguda possui um período de incubação de 4 a 14 dias, a partir da inoculação do parasita e uma duração de 2 a 4 meses. Por ser um período de clínica extremamente indefinida, pode ser caracterizada pela ausência de sintomas em $95 \%$ dos casos, ou ainda por sintomas que possam ser semelhantes a uma síndrome gripal leve (KRATZ MJ, et al., 2019).

Os $5 \%$ restantes dos casos podem apresentar sinais e sintomas relacionados ao local da inoculação ou manifestações sistêmicas (KRATZ JM, 2019; KRATZ JM, et al., 2018). Neste contexto, o diagnóstico é baseado, fundamentalmente, na identificação do parasita através da observação do sangue a fresco, esfregaço ou gota espessa do paciente infectado. Dentre essas variedades de testes, temos que o esfregaço possui uma menor sensibilidade quando comparado ao teste direto a fresco, sendo assim este torna-se o método de escolha mais recomendado para a realização do diagnóstico na fase aguda até o período de 30 dias (LIMA RS, et al., 2019).

Por outro lado, a fase crônica dessa patologia inicia-se 2 a 4 meses após a infecção aguda. Neste período, o parasita que antes era perceptível na corrente sanguínea torna-se menos evidente e apesar do paciente apresentar sorologia positiva as manifestações clínicas podem não ser significantes por um longo período. Entretanto, na forma crônica clássica da doença é comum o acometimento, misto ou isolado, do coração ou sistema digestório, gerando, dessa forma, a ocorrência de sintomas secundários decorrente do prejuízo orgânico que ocorre nesses órgãos (SCHMIDT MVS, et al., 2016).

Em virtude da ausência do parasita na circulação do indivíduo, o diagnóstico da fase crônica é baseado em um exame sorológico de alta sensibilidade e um de alta especificidade. Nessa perspectiva, temos por exemplo, o ELISA como uma técnica que detecta os anticorpos produzidos no corpo humano contra o parasita; a Hemaglutinação como um método simples e rápido que detecta os anti-T.cruzi através da ocorrência de uma reação de aglutinação das hemácias e a Imunofluorescência Indireta que através das suas reações padronizadas permite detectar a presença dos anticorpos em um grande número de amostras, sendo, portanto, amplamente utilizada nos serviços de saúde (ALVES DF, et al., 2018).

Os sinais e sintomas relacionados ao local da inoculação incluem: sinal de Romaña (edema indolor, endurado, pruriginoso, arroxeado, bipalpebral, unilateral e periocular que dificulta a abertura das pálpebras, 
causando secreção conjuntival limitada, dacrioadenite e adenopatia pré-auricular por satélite); e chagoma (nódulo subcutâneo elevado, eritematoso, edematoso, endurecido e moderadamente doloroso que é de aproximadamente $3 \mathrm{~cm}$ de diâmetro). Podem estar associados a linfonodos cervicais aumentados com aproximadamente $1 \mathrm{a} 2 \mathrm{~cm}$ de diâmetro, dolorosos à palpação, consistência suave, com bordas bem definidas e não aderidos a planos profundos (PÉREZ-MOLINA JA e MOLINA I, 2018).

Já as manifestações sistêmicas que podem ocorrer incluem: febre, astenia, adinamia, mialgia, artralgia, dor de cabeça, miocardite e hepatoesplenomegalia. A miocardite pode ocorrer com ou sem manifestações de comprometimento cardíaco, como taquicardia, ritmo galopar, prolongamento do intervalo PR e/ou QT, diminuição da tensão QRS, contrações ventriculares prematuras, bloqueio de ramo direito, alterações da onda T, pericardite, tamponamento cardíaco e insuficiência cardíaca (BENCK L, et al., 2018; BRAHMBHATT DH e COWIE MR, 2018).

Entretanto, o quadro clínico pode ser variado em determinados pacientes, como aqueles portadores de imunodepressão. Neste contexto, os indivíduos podem desenvolver um quadro mais agudo e grave da doença, como uma infecção mais intensa e comprometedora, lesões cardíacas graves que podem evoluir para um choque cardiogênico, meningoencefalite e acidente vascular cerebral (SOUZA DSC e POVOA RMS, 2016).

O tratamento da doença é sustentado por antiparasitários como benznidazol, agente derivado nitroimidazólico, e nifurtimox, composto nitrofurânico. Estes fármacos, apesar de possuírem um nível de evidência baixo, são amplamente utilizados, sendo o benznidazol o medicamento de primeira escolha, pois oferece poucos efeitos adversos ao indivíduo infectado, principalmente as crianças. A intolerância a essas substâncias é infrequente, porém quando ocorre as consequências mais comuns são a neuropatia, lesões cutâneas, anorexia, perda de peso, náuseas e vômitos. A administração de anti-histamínicos ou corticoides é recomendada quando há o aparecimento de lesões cutâneas e os anticonvulsivantes são a classe medicamentosa de escolha para o tratamento da neuropatia periférica (SOUZA DSC e POVOA RMS, 2016).

O desenvolvimento de vacinas para a prevenção da Doença de Chagas é uma alternativa promissora para o combate e erradicação dessa patologia. Atualmente, vacinas compostas com o antígeno alvo em todos os seus estágios (morto, atenuado, em frações subcelulares, dentro outros) são administradas na fase pré-clínica do estudo e utilizadas em cobaias como camundongos, cães e primatas não humanos. O objetivo dessas pesquisas é desenvolver uma vacina que possa promover uma resposta tanto terapêutica quanto profilática nos indivíduos imunizados (LUNA EJA e CAMPOS SRSLC, 2020).

A doença de chagas é menos comum fora das áreas rurais, onde os vetores são comumente encontrados em habitações rústicas. A sua disseminação ocorre por vetor, além de ser transmitida verticalmente entre mãe e feto ou pelo contato com fezes/urina contaminadas do inseto reduviidae (inseto triatomíneo, inseto do beijo) e, portanto, serve como hospedeiro intermediário do parasita. Outros modos de transmissão incluem transfusão de produtos sanguíneos, transplante de órgãos infectados ou consumo de alimentos ou bebidas infectadas. As principais complicações desta doença incluem cardiomegalia, doença gastrointestinal e, em alguns casos, neuropatia periférica (PINESI HT, et al., 2019).

Além de compreender a virulência do Trypanosoma cruzi, as suas formas de apresentação clínica e também as complicações causadas pela doença, é importante elucidar o perfil clínico e epidemiológico da doença. Rodrigues JRA, et al (2013) evidenciaram que entre os anos de 2007 e 2011 foram registrados no banco de dados do SINAN e FUNASA, somente no estado do Maranhão, 59,26\% e 62,16\% infectados do sexo masculino, demonstrando uma clara prevalência desta população.

Ademais, foi registrado ainda que a maior quantidade de casos é proveniente da zona rural atingindo idade entre 19 e 59 anos de idade. A principal forma de transmissão reconhecida no estudo foi a vetorial por via oral (RODRIGUES JR, et al., 2013). Cardoso EJS, et al (2016) traçaram o perfil epidemiológico da doença de chagas em uma cidade localizada no estado do Rio Grande do Sul. Observa-se que o perfil descrito corrobora com os resultados de outros estudos, evidenciando maior contaminação da população masculina, pacientes com baixa escolaridade, idade relativamente elevada e que desempenham funções em contato direto com a criação de animais e agricultura. 
O estudo registrou ainda que a desinformação é um fator de risco importante, pois desencadeia o manuseio incorreto do barbeiro e consequente aumento do potencial de infecção (CARDOSO EJS, et al., 2017). A prevalência da população rural infectada no Brasil pelo Trypanosoma cruzi pode ser justificada ainda por meio do comportamento humano, que tende a ocupar e explorar de forma desordenada o ambiente em que vive.

As baixas condições sociais, financeiras, educacionais e habitacionais, além do processo de destruição do ambiente natural, bem como, as migrações que ocorrem de maneira descontrolada, contribuem de forma significativa para o aumento do número de casos diagnosticados com doença de chagas. Por esse motivo, o desequilíbrio ecológico provocado por queimadas, corte de árvores e exploração de florestas às más condições de moradia, favorece a domiciliação dos insetos vetores (FERREIRA LF, et al., 2015).

Em estudo recente, Souza HP, et al (2020) apontaram que as regiões Norte, Centro-Oeste e sub-região meio-norte do Nordeste do Brasil possuem uma alta incidência de doenças infecto-parasitárias. Apesar da redução dos indicadores médios da pobreza, a concentração dessas patologias nesses territórios está atrelada a desigualdade socieconômica ainda presente no cenário nacional, o que, por sua vez, contribuiu para o adoecimento de grupos vulneráveis e afetados pela iniquidade estrutural histórica.

Afirmam ainda que a presença dessas patologias acaba servindo como um indicador de desenvolvimento regional, podendo, dessa forma, servir como um norteador para a construção de políticas públicas mais inclusivas que promover melhore assistência em saúde e condições de vida para as populações vulneráveis (SOUZA HP, et al., 2020).

Rosenthal LA, et al (2020) em pesquisa realizada com 132 pacientes internados no Hospital Escola da Universidade Federal de Pelotas, no Rio Grande do Sul, afirmam que uma população bem informada e com conhecimento adequado acerca dessa patologia e sua forma de transmissão contribuiria de forma significativa para o controle e prevenção dessa doença.

No entanto, os autores criticam as Secretarias Estaduais de Saúde, bem como o Ministério da Saúde, por possuírem poucas equipes que atuem promovendo educação neste contexto. Dessa forma, o papel dos profissionais de saúde inseridos na Atenção Básica como educadores torna-se essencial, visto que o compartilhamento de conhecimento sobre o tema com a população local é uma ferramenta relevante para o controle da endemia (ROSENTHAL LA, et al., 2020).

Partindo dessa perspectiva, Ferreira AM, et al (2018) conduziram um estudo que possuía como objetivo avaliar o conhecimento de 104 médicos da Atenção Primária à Saúde que atendiam em regiões endêmicas do norte de Minas Gerais e Vale do Jequitinhonha. Os autores apresentaram como resultado uma alta proporção, $49 \%$, de profissionais que declararam insuficiência de informações a respeito dessa patologia durante sua formação acadêmica, gerando, dessa forma, insegurança para o médico no atendimento e manejo de pacientes infectados pelo parasita. Os autores acreditam que tal situação ocorre em virtude de um ensino médico fragmentado e focado apenas no conhecimento hospitalar aliado a falta de educação permanente voltada ao (re)conhecimento da realidade dos territórios endêmicos (FERREIRA AM, et al., 2018).

Outrossim, é mister relatar ainda que a principal afecção associada a doenças de Chagas é a Hipertensão Arterial Sistêmica, seguida de outras doenças crônicas, como a Diabetes Mellitus. Silva MBA, et al, (2018) elucidaram que as complicações cardíacas são as mais prevalentes e também as mais graves, acometendo cerca de $30 \%$ a $40 \%$ dos pacientes diagnosticados.

É essencial destacar ainda que, os pesquisadores avaliaram 73 portadores de doença de chagas, e destes, $53,5 \%$ possui idade superior a 60 anos, $64,5 \%$ vivia com a renda menor que um salário mínimo e $60 \%$ é do sexo feminino, indo de encontro a outros estudos que registram a maior prevalência do sexo masculino (SILVA MBA, et al., 2018). De acordo com as estimativas da OMS, nos anos 90 , foi calculado que havia entre 16 e 18 milhões de pessoas infectadas, 100 milhões de pessoas em risco de contrair a doença e cerca de 45.000 mortes que poderiam ser atribuídas ao desenvolvimento de complicações. Nesse contexto, a Organização Pan-Americana da Saúde (OPAS) promoveu diferentes iniciativas para implementar e desenvolver medidas para controlar a transmissão da doença de Chagas por vetor, por transfusão e de mãe para feto, dependendo 
das características epidemiológicas de cada região (CONNERS EE, et al., 2016; PÉREZ-MOLINA JA e MOLINA I, 2018).

Como resultado dessas atividades, uma redução significativa na incidência da doença foi observada nos últimos anos. Estima-se que atualmente haja cerca de 6 a 8 milhões de pessoas infectadas, 65 a 100 milhões de pessoas em risco de contrair a doença e cerca de 12.000 mortes por ano que podem ser atribuídas ao desenvolvimento de complicações (PÉREZ-MOLINA JA e MOLINA I, 2018).

A maioria dessas pessoas está na América Latina, onde a doença é endêmica em 21 países. No entanto, movimentos de áreas rurais para áreas urbanas e migração internacional resultaram no afluxo de indivíduos infectados da América Latina para o resto do mundo, tornando a doença um problema para os sistemas de saúde em escala global (ÁLVAREZ-HERNÁNDEZ DA, et al, 2018; BENCK L, et al, 2018).

No Brasil, com as medidas de controle para eliminação do Triatoma infestans somados aos cuidados referentes a transmissão transfusional, houve uma significativa redução da incidência e da prevalência da doença de chagas. Apesar disso, a quantidade de infectados na região Nordeste brasileira ainda é alarmante, isso porque, o nordeste possui a maior quantidade de vetores secundários, como o Triatoma brasiliensis. Ademais, as péssimas condições de moradia que facilitam a reprodução dos triatomíneos, e ainda a ineficiência do controle epidemiológico potencializam a transmissão (ARRAIS FMA, et al., 2019; COOK CE e DÉCARY S, 2018).

Estudos de base populacional com enfoque em doenças negligenciadas evidenciaram o descuido que há com a doença de chagas. Nesse contexto, o geoprocessamento é uma importante ferramenta que permite a identificação, localização e padrão da doença nos grupos populacionais mais expostos (CARDOSO LP, et al., 2020).

Outrossim, os estudos de prevalência da transmissão chagásica entre populações doadoras de sangue são considerados muito interessantes no registro de casos na forma indeterminada da doença. Isto reflete que a Doença de Chagas é clinicamente silenciosa e denunciada pela presença de anticorpos antiTrypanossoma cruzi circulantes (SANTANA MP, et al., 2018). Desta maneira, o doador sanguíneo que possui em seu soro a presença dos anticorpos anti-Trypanossoma torna-se impossibilitado de realizar doações posteriores e a bolsa coletada é direcionada para descarte. É necessário ainda que, os pacientes soro positivos sejam encaminhados para a avaliação médica para que sejam realizados testes complementares com o intuito de confirmar o diagnóstico da doença de chagas e submetê-lo às orientações cabíveis (BRUTTI B, et al., 2019).

\section{CONSIDERAÇÕES FINAIS}

O perfil epidemiológico dos pacientes portadores da doença de chagas ocorre de forma preferencial em populações do sexo masculino, que residem em zona rural. Ademais, observou-se ainda uma maior prevalência de infecção na faixa etária entre 19 a 59 anos de idade. É indubitável ainda, a relação entre os portadores da doença com as baixas condições socioeconômicas, migração, baixa escolaridade e condições de moradia precária. Salientamos a importância do processo de educação em saúde, para informar a população acerca da transmissão da doença e dos cuidados necessários referentes a prevenção. Ainda, é necessário que novos estudos sejam realizados, especialmente em pacientes idosos maiores de 60 anos de idade, tendo em vista a maior importância da infecção nesta faixa etária associada a comorbidades.

\section{REFERÊNCIAS}

1. ÁLVAREZ-HERNÁNDEZ DA, et al. Chagas disease: Current perspectives on a forgotten disease. Revista Médica del Hospital General de México, 2018; 81 (3): 154-164.

2. ALVES DF, et al. Métodos de diagnóstico para a doença de Chagas: uma atualização Diagnostic methods of Chagas disease: an update. Revista brasileira de analálise Clinica, 2019; 50(4): 330-333

3. ARRAIS FMA, et al. Perfil entomológico da doença de Chagas no município de Potengi - CE, Brasil. Saúde, 2019; 45 (1): 1-13. 
4. BENCK L, et al. Jignesh. Diagnosis and Management of Chagas Cardiomyopathy in the United States. Current Cardiology Reports, 2018; 20 (12).

5. BRAHMBHATT DH, COWIE MR. Heart failure: classification and pathophysiology. Medicine (United Kingdom), 2018; 46 (10): 587-593.

6. BRUTTI B, et al. Diabete Mellitus: definição, diagnóstico, tratamento e mortalidade no Brasil, Rio Grande do Sul e Santa Maria, no período de 2010 a 2014. Brazilian Journal of Health Review, 2019; 2 (4): 3174-3182.

7. BRASIL. Ministério da Saúde do Brasil. Protocolo Clínico e Diretrizes Terapêuticas Doença de Chagas. CONITEC, $2018 ; 1-144$.

8. CARDOSO EJ, et al. Perfil epidemiológico dos portadores de doença de chagas: dos indicadores de risco ao processo de enfrentamento da doença. Arq. Ciênc. Saúde, 2017; 24 (1): 41-46.

9. CARDOSO LP, et al. Spatial distribution of Chagas disease and its correlation with health services. Revista escola enfermagem USP, 2020; 54: 1-8.

10. CAVALCANTI MAF, et al. Manifestations and strategies of coping with chagas disease that interfere in the quality of life of the individual: A systematic review. Ciencia e Saude Coletiva, 2019; 24 (4): 1405-1416.

11. CONNERS EE, et al. A global systematic review of Chagas disease prevalence among migrants. Acta Tropica, 2016; 156: 68-78.

12. COOK CE, DÉCARY S. Doença de chagas: tendência epidemiológica por regiões do Brasil. Brazilian Journal of Physical Therapy, 2018; 1 (1): 252-259.

13. COSTA AC, et al. Satisfaction of Chagas disease patients attended at a pharmaceutical care service in the State of Ceará, Brazil. Ciencia e Saude Coletiva, 2018; 23 (5); 1483-1494.

14. DIAS JCP, et al. II Consenso Brasileiro em Doença de Chagas, 2015. Epidemiologia e servicos de saude : revista do Sistema Unico de Saude do Brasil, 2016; 25; 7-86.

15. FERREIRA LF, et al. Perfil epidemiológico dos idosos chagásicos da Unidade de Saúde da Família do bairro Nossa Senhora de Fátima, USF - Nossa Senhora de Fátima, da cidade de Paracatu - MG. Rev Med, São Paulo, $2015 ; 94$ (2); 120-125.

16. FERREIRA AM, et al. Avaliação Do Conhecimento Acerca Do Manejo Clínico De Portadores Da Doença De Chagas Em Região Endêmica No Brasil. Revista APS, 2018; 21(3): 345 - 354

17. GRAYSON M. Chagas disease, 2010; 465.

18. HABERLAND A, et al. Chronic Chagas disease: From basics to laboratory medicine. Clinical Chemistry and Laboratory Medicine, 2013; 51 (2): 271-294.

19. LIMA RS, et al. Chagas disease: a bibliographic update. Revista brasileira de análise clínica, 2019; 51(2): 103-106

20. LUNA EJA, CAMPOS SRSLC. O desenvolvimento de vacinas contra as doenças tropicais negligenciadas. Caderno de Saúde Pública, 2020; 36(2):1-14.

21. AGARAKIS M, et al. Cardiac surgery for Chagas disease. Journal of Cardiac Surgery, 2018; 33 (10); $597-602$.

22. MARTINS-MELO FR, et al. Multiple causes of death related to Chagas' disease in Brazil, 1999 to 2007. Revista da Sociedade Brasileira de Medicina Tropical, 2012; 45 (5); 591-596.

23. KRATZ MJ. Drug discovery for chagas disease: A viewpoint. Acta Tropica, 2019; 198.

24. KRATZ MJ, et al. Clinical and pharmacological profile of benznidazole for treatment of Chagas disease. Expert Review of Clinical Pharmacology, 2018; 11 (10); 943-957.

25. ORTIZ JV, et al. Cardiac Evaluation in the Acute Phase of Chagas' Disease with Post-Treatment Evolution in Patients Attended in the State of Amazonas, Brazil. Arquivos Brasileiros de Cardiologia, 2018; 112 (3); 240-246.

26. PEREIRA LS, et al. Perfil clínico e epidemiológico de pacientes idosos com doença de Chagas atendidos entre 20052013 por um serviço de atenção farmacêutica no estado do Ceará, nordeste do Brasil. Revista do Instituto de Medicina Tropical de Sao Paulo, 2015; 57 (2); 145-152.

27. PÉREZ-MOLINA JA, MOLINA I. Chagas disease. The Lancet, 2018; 391 (10115); $82-94$.

28. PÉREZ-MOLINA JA, MOLINA I. Chagas disease cardiomyopathy treatment remains a challenge - Authors' reply. The Lancet, 2018; 391 (10136); 2209-2210.

29. PINESI HT, et al. Caso 4/2019- Homem de 26 anos com doença de chagas congênita e transplante cardíaco. Arquivos brasileiros de cardiologia, 2019; 113(2):286-293.

30. RODRIGUES JR, et al. Doença de Chagas aguda no estado do Maranhão, Brasil: uma comparação entre os bancos de dados do SINAN e da FUNASA. J Manag Prim Health Care, 2013; 4 (1): 3-9.

31. ROSENTHAL LA, et al. Conhecimentos sobre a doença de Chagas e seus vetores em habitantes de área endêmica do Rio Grande do Sul, Brasil. Caderno de saúde coletiva, 2020; 28(3): 345-352.

32. SANTANA MP, et al. Prevalência da doença de chagas entre doadores de sangue do estado do Piauí, Brasil, no período de 2004 a 2013. Cadernos de Saude Publica, 2018; 34 (2): 1-15.

33. SCHMIDT MVS, et al. Aspectos imunológicos da infecção por Trypanosoma cruzi: Persistência do parasite e autoimunidade. Revista sociedade de cardiologia do Estado de Säo Paulo, 2016; 26(4): 240-245.

34. SILVA MBA, et al. Doença de Chagas: Conhecimento dos portadores atendidos em Centro de Referência de Pernambuco. Revista Enfermagem Digital Cuidado e Promoção da Saúde, São Paulo, 2018; 3 (2): 120-125.

35. SOUZA DSC, POVOA RMS. Aspectos epidemiológicos e clínicos da Doença de Chagas aguda no Brasil e na américa Latina. Revista sociedade de cardiologia do estado de Säo Paulo, 2016; 26(4): 222-229

36. SOUZA HP, et al. Doenças infecciosas e parasitárias no Brasil de 2010 a 2017: aspectos para vigilância em saúde. Revista Panamericana de Salud Publica, 2020; 44: 1-7.

37. VELOSO HH, et al. Chagas heart disease in professional athletes from endemic countries: A notorious case calls attention for its identification and stratification. International Journal of Cardiology, 2016; 207: 115-116. 